\title{
Identification of active molecules against Mycobacterial Shikimate Kinase from Chemical library and their affinity with different domains
}

\author{
Sapna Pandey ${ }^{1}$, Ekta Dhamija ${ }^{1}$, Sanjay Kumar ${ }^{1}$, Pragya Yadav $^{1}$, Tadigopula Narender ${ }^{1}$, \\ Arnava Dasgupta ${ }^{1}$, Ravishankar Ramachandran ${ }^{1}$, and KISHORE SRIVASTAVA ${ }^{1}$ \\ ${ }^{1}$ Central Drug Research Institute
}

November 9, 2020

\begin{abstract}
Tuberculosis (TB), regardless of being the oldest disease is still a menace that humans have not been able to control. With the advancement in the drug discovery programme, target-based drug discovery appears to be one of the promising techniques for the development of future therapeutics. It involves identifying an essential gene involved in the pathogenesis of the disease and then targeting the protein against a defined chemical library. Shikimate kinase is one such validated target in mycobacterium. It is vital for the growth of bacteria and is absent in mammals, making it an ideal drug target. Here 6427 compounds were screened through structure based virtual screening where compound S-014-1049 was found active against H37Rv and proven non-cytotoxic in in vitro studies. It specifically binds to the core domain of MTSK.
\end{abstract}

\section{Introduction}

The development of drug resistance is a severe threat to public health. Presently, use of antibiotics targets bacterial structure and essential functions. Due to the well-identified anti-mycobacterial resistance, there has been an increased interest in the discovery of novel drugs to target other essential processes of bacterial survival. Currently, target-based drug screening is promising and efficient way for development of therapeutic agents. In recent years, extensive efforts have been made for the discovery of inhibitors of enzymes involved in the biosynthesis of aromatic amino acids. One such well-recognised pathway is shikimic acid pathway consisting of seven enzymatic reactions responsible for the production of chorismate; a precursor for essential amino acids. Among seven enzymes, the most valuable target is Shikimate Kinase (SK). Inhibition of this enzyme is fatal for M. tuberculosis $(M t b) .{ }^{1}$ SK inhibitors offer potential for development of new antitubercular drugs that are selective for $M t b$ since mammals do not have Shikimate pathway enzymes necessary for de novo synthesis of essential amino acids. MTSK belongs to the family of nucleoside monophosphate (NMP) kinases and consist of 3 domains: LID (LD), CORE (CD), and substrate binding (SBD) domains. ${ }^{2,} 3$

Owing to the importance of this enzyme, structural based virtual screening of compounds from institute library was done where S-014-1049 was found to be active against SK and against the mycobacteria in culture as well. This compound specifically binds to the CD of the enzyme which was primarily confirmed during the docking studies and further by validating with the mutants.It is a polyphenol and derivative of curcumin. In some recent studies a few polyphenols were found active against $M t b$ which also have many health benefits. ${ }^{4}$ The biological and pharmacological properties make polyphenols a valuable druggable molecule.$^{5,6}$ In one of our previous studies, we found an existing polyphenolic PKC- $\delta$ inhibitor, Rottlerin to be active against mycobacteria where it significantly inhibited SK activity and has been used as positive control in this study. ${ }^{7}$ Therefore, compound S-014-1049 could be a promising drug candidate for further development 


\section{Materials and methods}

\section{Cloning and expression of recombinantSK}

ORF of the gene (Rv2539; MSMEG 3031) was PCR amplified using genomic DNA of H37Rv and M.smegmatis and then ligated into TA vector. Further, the gene was sub-cloned in pET28a (In vitrogen) expression vector and introduced intoE.coli BL21 (DE3). For expression of Rv2539 (MTSK) and MSMEG 3031 (MSSK), cultures were induced with $1.0 \mathrm{mM}$ IPTG at $18^{\circ} \mathrm{C}$, and $0.8 \mathrm{mM}$ IPTG at $22^{\circ} \mathrm{C}$ respectively. The expressed proteins were purified through Ni-NTA column (Qiagen).

\section{Enzyme assay and anti-mycobacterial screening}

The enzymatic activity of MTSK was assessed through ADP-Glo kinase assay as mentioned the previous study. ${ }^{7}$ For inhibition assay, MTSK was incubated at different concentrations $(12.5 \mu \mathrm{g} / \mathrm{ml}$ to $50 \mu \mathrm{g} / \mathrm{ml})$ of compounds for an hour at $37^{\circ} \mathrm{C}$, subsequently followed by addition of $2 \mu \mathrm{g} / \mathrm{ml}$ shikimic acid. After completion of overall reaction, phosphorylation of substrate by the kinase was measured by luminometer in terms of relative luminescence unit (RLU).Anti-mycobacterial screening was done using MABA and MGIT according to our previous study. ${ }^{7,8}$

\section{Site directed mutagenesis (SDM)}

The mutants of SK were created using a variant PCR where the whole plasmid DNA is amplified using overlapping primers running in opposite directions. pET28a :SK plasmid was used as template to create phospho-null mutants, as described earlier. ${ }^{8}$

\section{Results}

\section{Structure based virtual and in vitroscreening of active compounds}

In our study, structure based virtual screening of 6427 compounds generated 134 hits against SK. Of these, 44 compounds with good binding energy were analyzed by in vitro studies. Of these, 44 compounds with good binding energy were analyzed by in vitro studies (Figure 1A). MABA and MGIT were used for the in vitro study against $M t b$ using protocol described earlier. ${ }^{8}$ Of all 44 compounds seven exhibiting $>55 \%$ inhibition at $25 \mu \mathrm{g} / \mathrm{ml}$ (table 1 ) and four were active against H37Rv at [?]3.12 $\mu \mathrm{g} / \mathrm{ml}$ (table 2). The docking studies also showed that all the four hits also have active binding affinity with SK (Figure 1B). The most interacted residues include Glycine, Serine, Isoleucine, Aspartate, Arginine and Alanine, which are present in active region and form hydrogen bond with each hit. The compounds were evaluated for their cytotoxicity in Vero/THP1 cells at varying compound concentrations using MABA. This deciphered that S-014-1049 is non-cytotoxic to the cells with selective index of more than 16. Compounds with selective index $>10$ are appropriate drug candidates (Table 2).

\section{Specific interaction study of complex between S-014-1049 and SK using bioinformatics tool}

S-014-1049 was found to be non-toxic to the vero cells. Complex study of S-014-1049 with SK was done to check the specific binding of this compound to different domains of the enzyme. The binding result showed residues GLY14, LYS15, SER16, THR17, and ARG117 in binding region of the compound. LYS15 forms hydrogen bond with fluorine atom (F3-N) of the ligand at $3.2 \AA$. Similarly, nearby residues such as GLY14, THR17 and SER16 also make hydrogen bond with fluorine atoms (F1, F2 and F3). ARG117 also forms hydrogen bond with fluorine atom. ASP34 form pi-anion interaction with $3.84 \AA$ distance which is an unfavourable interaction. The other residues which come in binding region are PRO118, LEU119 and PHE49, these residues form hydrophobic interaction with ligand at $>4 \AA$ distance. From the study we can conclude that this compound interacts via strong hydrogen bond with several residues of CD as well as with one residue (ARG117) of LD (Figure 1C).

\section{Kinase activity of SKagainstS-014-1049}

Kinase activity of MTSK was checked against S-014-1049 at varying concentrations using ADP-Glo assay, and rottlerin was used as a positive control for the assay. Outcome of the result showed $>50 \%$ inhibition at 
$50 \mu \mathrm{g} / \mathrm{ml}$ concentration (Figure 1D)

\section{Effect of S-014-1049 on Ra-wt at different concentrations}

Log phase culture of Ra-wt was taken and treated with S-014-1049 and rottlerin at $1 \mu \mathrm{g} / \mathrm{ml}, 2 \mu \mathrm{g} / \mathrm{ml}$ and $4 \mu \mathrm{g} / \mathrm{ml}$ for an hour with constant shaking. After treatment lysates of the culture were prepared and analysed using western blotting. Maximum inhibition of Ra-wt was observed at 2X MIC concentration of S-014-1049 whereas rottlerin showed significant inhibition at all concentrations (Figure 1E, F).

\section{Comparative domain analysis of SK of different mycobacterial strains}

Clustal Omega pairwise sequence alignment software was used to analyse the domains of SK of H37Rv and M.smegmatis (MS). It was observed that CD was the only domain which is $100 \%$ conserved in both the strains (Figure 2A). Using MABA MIC ofS-014-1049was calculated against H37Rv, H37Ra and MS. The three strains showed inhibition at a concentration of $3.12 \mu \mathrm{g} / \mathrm{ml}, 12.5 \mu \mathrm{g} / \mathrm{ml}$ and $25 \mu \mathrm{g} / \mathrm{ml} \mathrm{respectively} \mathrm{(Figure}$ 2B). Since, all the three strains have a conserved CD and are inhibited by the compound which possibly suggests thatS-014-1049 binds to CD of SK.

\section{Analysis of kinase activity of mutant proteins}

Kinase activity of the mutant proteins was assessed to check the most essential domain for the activity of SK using ADP-Glo kinase assay. Assay was performed using $2 \mu \mathrm{g} / \mathrm{ml}$ of shikimic acid and $600 \mathrm{ng}$ of purified protein. Comparative analysis of the kinase activity of mutant proteins was done with respect to purified recombinant SK. The result shows $>90 \%$ decrease in activity of protein where CD was mutated $\left(\mathrm{CD}^{15}: \mathrm{SK}\right)$ while, around $50 \%$ decrease was observed in the protein where LD was manipulated (LD $\left.{ }^{117}: \mathrm{SK}\right)$. Moreover, no effect was observed in kinase activity of the protein where SBD was mutated (SBD $\left.{ }^{34}: \mathrm{SK}\right)$. Overall findings suggest that the activity of the enzyme decreases significantly upon mutation of $\mathrm{CD}$, depicting its vital role in enzyme activity whereas $\mathrm{SBD}^{34}$ :SK does not have any effect on enzymatic activity (Figure 2D).

\section{Inhibitory profiling of S-014-1049 against mutant SK}

Comparative study of the kinase activity of the 3 mutants $\left(\mathrm{CD}^{15}\right.$ :SK, $\mathrm{SBD}^{34}$ :SK, and $\mathrm{LD}^{117}$ :SK) of SK using ADP-Glo kinase assay and wild type MTSK was used as a control. Significant inhibition of enzyme activity was observed in $\mathrm{SBD}^{34}: \mathrm{SK}, \mathrm{LD}^{117}$ :SK and MTSK compared to $\mathrm{CD}^{15}: \mathrm{SK}$. Hence, it can be inferred that the binding of the compound with the protein, occurs in case of intact CD which influences in the decrease of enzyme activity (Figure 2E).

\section{Discussion}

Most serious public health problem of last decade has been due to the increasing resistance to clinically available useful antibiotics resulting in prevalence of tuberculosis. ${ }^{9}$ Thus, there is an urgent necessity to look for better anti-bacterials for which the new drug targets need to be identified. Against which a large pool of compound library can be screened using structure-based drug screening approach. Of late, an extensively evaluated bacterial target for the development of drugs is the protein of shikimate pathway. The fifth enzyme of the pathway, SK plays fundamental role in viability of microorganism. Due to absence of its homolog in mammals, it can serve as a promising target for therapeutic intervention.

Orthologs of SK are present in all the $M t b$ complex bacteria. Figure $2 \mathrm{~A}$ shows pairwise alignment of proteins among the three domains of SK namely CD, SBD and LD between $M t b$ and MS. CD is the only domain which is conserved in $M t b$ and in MS whereas SBD and LD are semi-conserved.

In the current study to find the specific inhibitor for SK, structure based virtual screening of the in-house library was achieved. Out of which 44 hits had the highest docking score and were further evaluated in in vitro study against $M t b$ using MABA and MGIT. The result indicated 4 compounds to be active against $M t b$ . Specific activity of these 4 compounds against SK was assessed through ADP-Glo kinase assay where S-0140149 was found to be the most-inhibitory compound. Cytoxicity of the four compounds was also checked in THP-1 human macrophages and the only compound which was found to be non-toxic was S-014-1049 
(Table 2). In previous study from our group ${ }^{10}$ this compound was already found active against drug resistant and drug susceptible $M t b$ strains and also shown synergistic effect with first line drugs of tuberculosis. In the same study ${ }^{10}$ this compound shown reduction in cfu of murine model infected with $M t b$. Efficacy of this compound was checked against H37Ra at different concentrations of MIC (0.5X, $1 \mathrm{X}$ and 2X) using rottlerin as a positive control. $>50 \%$ inhibition of expression was observed at all the three concentrations in western blot assay (Figure 1E).

The 3 domains of SK were analysed by creating mutants at important residue in each domain. Assessing the kinase activity of the three mutants demonstrated that CD is most important among the three in defining the activity. Activity of compound S-014-1049 was also checked against the mutant proteins using ADP-Glo assay and the result showed specific binding of this compound to the CD. Docking study of the complex between SK and S-014-1049 also showed that LYS15 residue of CD forms hydrogen bond with fluorine atom (F3-N) of ligand at $3.2 \AA$ and nearby residues such as GLY14, THR17 and SER16 also form hydrogen bond with fluorine atoms (F1, F2 and F3). ARG117 also makes hydrogen bond with fluorine atom as well as pi-cation interaction with benzene ring. ASP34 forms pi-anion interaction with $3.84 \AA$ distance (Figure 1C). Hence, both in vitro and docking studies indicate binding of S-014-1049 to CD of SK Compound S-014-1049 is a polyphenol and derivative of curcumin. In some of the recent studies few of the polyphenols were found to be active against $M t b$ and are known to have many health benefits. The biological and pharmacological properties make polyphenols a valuable druggable molecule. ${ }^{4,5}$. Therefore, this compound can be a promising drug candidate in future to cope with AMR.

\section{Acknowledgement}

We thank Director, CSIR-CDRI for the support. The work has been funded through WOS-A (DST) and by the CSIR-CDRI grant MLP0120. The CSIR-CDRI communication number allotted to this manuscript is (to be included after acceptance).

\section{Figure legends}

Figure 1 : Virtual screening work flow of in house library compounds (A) 3D representation of 4 hits (which were found active against $\mathrm{H} 37 \mathrm{Rv}$ in in vitro studies) and interaction with SK protein along with 2D view of individual compound. Cyan colour ribbon like structure is SK, and magenta color structure represent compounds (B). S-014-1049 was found to be non-cytotoxic to the cells, and its interaction by different types of bonds with specific residue of SK. Where most favorable bond (hydrogen bond) is represented with green (C) Inhibitory profile of the compounds against purified SK using ADP Glo kinase assay at three different concentrations (D) Inhibitory action of S-014-1049 and rottlerin on Ra-wt at 0.5X, 1X, and 2X MIC. The effect was observed after 1 hour of drug treatment where hsp 65 was taken as a control. Lane 1 is no drug and lanes 2, 3, 4 are $0.5 \mathrm{X}, 1 \mathrm{X}, 2 \mathrm{X}$ of MIC $(0.2 \mu \mathrm{g} / \mu \mathrm{l})$ of the compound (S-014-1049) respectively. Lanes 5, 6,7 are $0.5 \mathrm{X}, 1 \mathrm{X}, 2 \mathrm{X}$ of $\mathrm{MIC}$ concentration of rottlerin (E) Blot was analysed by densitometry where $\mathrm{AU}$ is arbitrary unit(F)

Figure 2. Comparative domain analysis of SK of $M t b$ and MSby using bioinformatic tool (A) Inhibition profile of S-014-1049 against three strains of mycobacteria using MABA (B) Overexpression of mutant protein (a) in BL21(DE3) using $1.0 \mathrm{mM}$ IPTG concentration. Lane 1 is uninduced, lane 2 is mutant of CD $\left(\mathrm{CD}^{15}: \mathrm{SK}\right)$, lane 3 is mutant of SBD $\left(\mathrm{SBD}^{34}: \mathrm{SK}\right)$ and lane 4 is mutant of $\mathrm{LD}\left(\mathrm{LD}^{117}: \mathrm{SK}\right)$. Purification of the mutant protein through Ni-NTA chromatography (b) lane 1 is mutant of CD (CD $\left.{ }^{15}: \mathrm{SK}\right)$, lane 2 is mutant of SBD $\left(\mathrm{SBD}^{34}: \mathrm{SK}\right)$ and lane 3 is mutant of LD (LD $\left.{ }^{117}: \mathrm{SK}\right)$ respectively (C) Kinase assay of the mutant purified protein in comparison to the recombinant purified SK, where pink colour bar is for purified SK, green is for $\mathrm{SBD}^{34}$ : SK, red is for $\mathrm{CD}^{15}$ : SK and yellow is for $\mathrm{LD}^{117}$ : SK(D). Effect of compound S-014-1049 at $50 \mu \mathrm{g} / \mathrm{ml}$ concentration against wt SK and mutant SKs at 600ng protein concentration using ADP-Glo kinase assay (E).

\section{References}

[1] Parish T, Stoker NG. The common aromatic amino acid biosynthesis pathway is essential in Mycobac- 
terium tuberculosis. Microbiology 2002;148:3069-77.

[2] Oliveira JS, Pinto CA, Basso LA, Santos DS. Cloning and overexpression in soluble form of functional shikimate kinase and 5-enolpyruvylshikimate 3-phosphate synthase enzymes from Mycobacterium tuberculosis. Protein Expr Purif 2001;22:430-435

[3] Simithy J, Gill G, Wang Y, Goodwin DC, Calderón AI. Development of an ESI-LC-MS-based assay for kinetic evaluation of mycobacterium tuberculosis shikimate kinase activity and inhibition. Anal Chem $2015 ; 87: 2129-36$.

[4] Tachibana H. Green tea polyphenol sensing. Proc Jpn Acad Ser B Phys Biol Sci 2011;87:66-80.

[5] Velayutham P, Babu A, Liu D, Gilbert ER. Recent advances in understanding the anti-diabetic actions of dietary flavonoids n.d. J Nutr Biochem 2013;24(11):1777-1789

[6] Gibbons S. Phytochemicals for bacterial resistance - Strengths, weaknesses and opportunities. Planta Med., 2008;74(6):594-602 https://doi.org/10.1055/s-2008-1074518.

[7] Pandey S, Chatterjee A, Jaiswal S, Kumar S, Ramachandran R, Srivastava KK. Protein kinase C$\Delta$ inhibitor, Rottlerin inhibits growth and survival of mycobacteria exclusively through Shikimate kinase. Biochem Biophys Res Commun 2016;478:721-6.

[8] Chatterjee A, Pandey S, Singh PK, Pathak NP, Rai N, Ramachandran R, et al. Biochemical and functional characterizations of tyrosine phosphatases from pathogenic and nonpathogenic mycobacteria: indication of phenyl cyclopropyl methyl-/phenyl butenyl azoles as tyrosine phosphatase inhibitors. Appl Microbiol Biotechnol 2015;99:7539-48.

[9] Mehta S, Mehta SS, Thokchom SK, Patyal P. A critical insight into shikimate kinase pathway. Innovare Academic Sciences 2015;7:25-7.

[10] Singh AK, Yadav P, Karaulia P, Singh VK, Gupta P, Puttrevu SK, et al. Biological evaluation of novel curcumin-pyrazole-mannich derivative active against drug-resistant Mycobacterium tuberculosis. Future Microbiol 2017.

\section{Hosted file}

Table 1.pdf available at https://authorea.com/users/374103/articles/491726-identificationof-active-molecules-against-mycobacterial-shikimate-kinase-from-chemical-library-andtheir-affinity-with-different-domains

\section{Hosted file}

Table 2.pdf available at https://authorea.com/users/374103/articles/491726-identificationof-active-molecules-against-mycobacterial-shikimate-kinase-from-chemical-library-andtheir-affinity-with-different-domains 


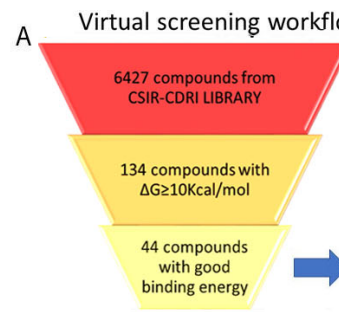

B

c

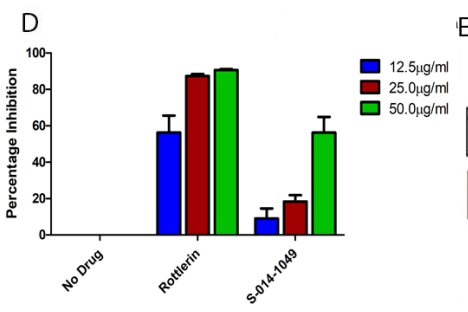

E

F
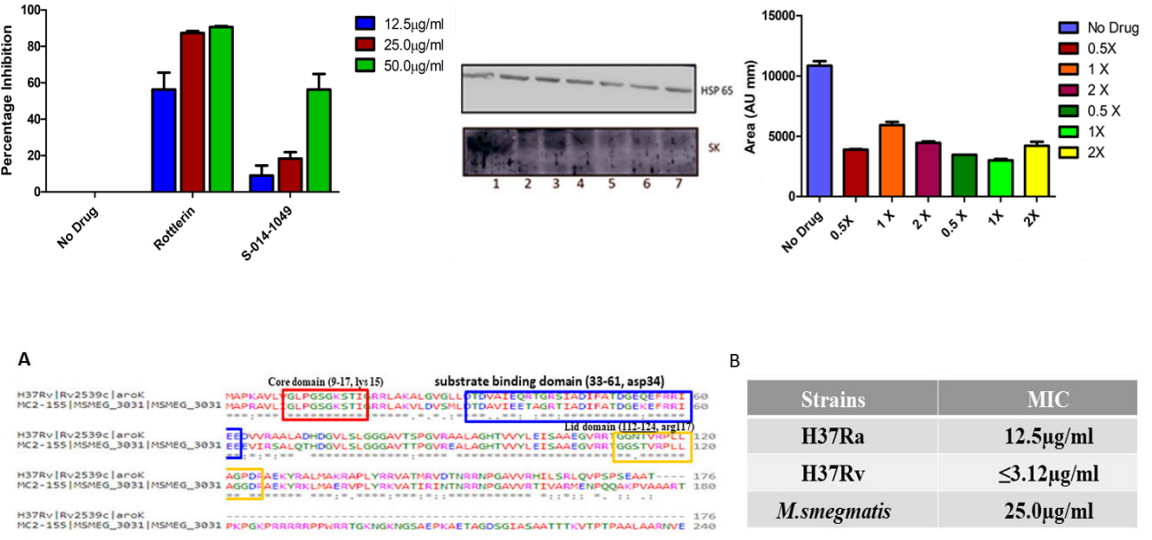

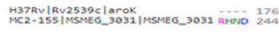

c

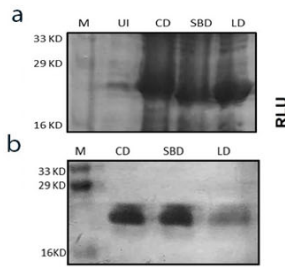

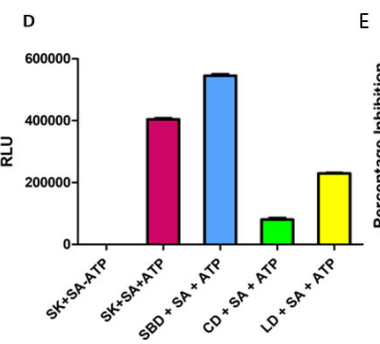

E

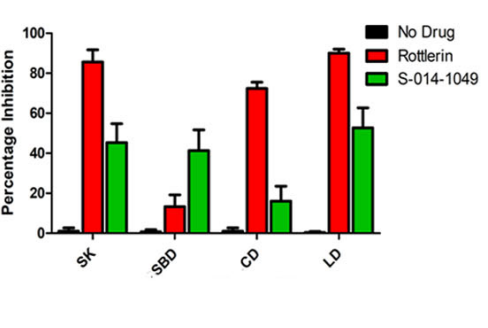

